OPEN ACCESS

Edited by:

Ryogo Minamimoto,

National Center For Global Health and

Medicine, Japan

Reviewed by:

Liyong Ma,

Harbin Institute of Technology, China

Cyril Jaudet,

Centre François Baclesse, France

*Correspondence:

Xiaobo Yang

yangx@gxmu.edu.cn

${ }^{t}$ These authors have contributed equally to this work and

share first authorship

Specialty section:

This article was submitted to

Cancer Imaging and Image-directed Interventions,

a section of the journal

Frontiers in Oncology

Received: 22 August 2021 Accepted: 06 October 2021 Published: 28 October 2021

Citation:

Guo Y, Xu J, Li X, Zheng L, Pan W, Qiu M, Mao $S$, Huang $D$ and Yang $X$ (2021) Classification and Diagnosis of

Residual Thyroid Tissue in SPECT Images Based on Fine-Tuning Deep Convolutional Neural Network.

Front. Oncol. 11:762643.

doi: 10.3389/fonc.2021.762643

\section{Classification and Diagnosis of Residual Thyroid Tissue in SPECT Images Based on Fine-Tuning Deep Convolutional Neural Network}

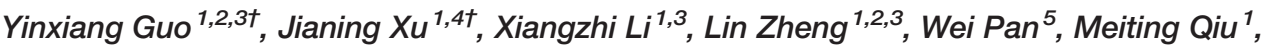 \\ Shuyi Mao ${ }^{5}$, Dongfei Huang ${ }^{5}$ and Xiaobo Yang ${ }^{1,3 *}$
}

${ }^{1}$ Guangxi Key Laboratory on Precise Prevention and Treatment for Thyroid Tumor, The Second Affiliated Hospital, Guangxi University of Science and Technology, Liuzhou, China, ${ }^{2}$ School of Science, Guangxi University of Science and Technology, Liuzhou, China, ${ }^{3}$ Department of Public Health, School of Medicine, Guangxi University of Science and Technology, Liuzhou, China, ${ }^{4}$ School of Microelectronics and Materials Engineering, Guangxi University of Science and Technology, Liuzhou, China, ${ }^{5}$ Department of Nuclear Medicine, The Second Affiliated Hospital of Guangxi University of Science and Technology, Liuzhou, China

Patients with thyroid cancer will take a small dose of ${ }^{131} \mathrm{I}$ after undergoing a total thyroidectomy. Single-photon emission computed tomography (SPECT) is used to diagnose whether thyroid tissue remains in the body. However, it is difficult for human eyes to observe the specificity of SPECT images in different categories, and it is difficult for doctors to accurately diagnose the residual thyroid tissue in patients based on SPECT images. At present, the research on the classification of thyroid tissue residues after thyroidectomy is still in a blank state. This paper proposes a ResNet-18 fine-tuning method based on the convolutional neural network model. First, preprocess the SPECT images to improve the image quality and remove background interference. Secondly, use the preprocessed image samples to fine-tune the pretrained ResNet-18 model to obtain better features and finally use the Softmax classifier to diagnose the residual thyroid tissue. The method has been tested on SPECT images of 446 patients collected by local hospital and compared with the widely used lightweight network SqueezeNet model and ShuffleNetV2 model. Due to the small data set, this paper conducted 10 random grouping experiments. Each experiment divided the data set into training set and test set at a ratio of 3:1. The accuracy and sensitivity rates of the model proposed in this paper are $96.69 \%$ and $94.75 \%$, which are significantly higher than other models $(p<0.05)$. The specificity and precision rates are $99.6 \%$ and $99.96 \%$, respectively, and there is no significant difference compared with other models. ( $p>0.05)$. The area under the curve of the proposed model, SqueezeNet, and ShuffleNetv2 are 0.988 (95\% Cl, 0.941-1.000), $0.898(95 \% \mathrm{Cl}, 0.819-0.951)(p=0.0257)$, and $0.885(95 \% \mathrm{Cl}, 0.803-0.941)(p=0.0057)$ 
$(p<0.05)$. We prove that this thyroid tissue residue classification system can be used as a computer-aided diagnosis method to effectively improve the diagnostic accuracy of thyroid tissue residues. While more accurately diagnosing patients with residual thyroid tissue in the body, we try our best to avoid the occurrence of overtreatment, which reflects its potential clinical application value.

Keywords: SPECT image, thyroid cancer, deep learning, fine-tuning, classification diagnosis, overtreatment

\section{INTRODUCTION}

Thyroid nodules are irregular masses around the thyroid gland and are common diseases of the endocrine system. Thyroid nodules can be divided into benign and malignant. According to the global cancer statistics report, the incidence of malignant thyroid nodules, that is, thyroid cancer, ranks first in endocrine malignant tumors. Total thyroidectomy is the most commonly used clinical surgery to treat thyroid cancer. Single-photon emission computed tomography (SPECT) is one of the representative techniques of nuclear medicine imaging (1). Compared with general medical imaging technology, SPECT can not only display the morphology and structure of tissues and organs, but more importantly, it can provide some characteristic information of tissues and cells at the molecular level, thereby showing the metabolic status of tissues and organs. In 2020, the total number of single-photon imaging inspections in China was more than 2.5 million (2). At this stage, it is mainly based on SPECT images to determine whether there is thyroid tissue remaining in the body after ablation of thyroid cancer patients. During the ablation treatment of thyroid cancer patients, SPECT images can dynamically observe the effect before and after treatment and the metastasis of the lesion from the pathological point of view (3). Therefore, SPECT is widely used in clinical diagnosis in ${ }^{131} \mathrm{I}$ ablative treatment with its unique advantages $(4,5)$.

In 2015, the "Guidelines for the Diagnosis and Treatment of Adult Thyroid Nodules and Differentiated Thyroid Cancer" issued by the American Thyroid Association (ATA) pointed out that in order to reduce unnecessary radiation damage and avoid overtreatment problems, it is recommended to use $30 \mathrm{mCi}$ for ${ }^{131} \mathrm{I}$ ablative treatment for middle and low-risk patients (6). In clinical practice, $2-5 \mathrm{mCi}$ is generally used for the detection of residual thyroid tissue after total thyroidectomy (7). Because of the dose of $30 \mathrm{mCi}$ which is close to the dose for one treatment purpose and is not used for diagnosis, which may cause patients to develop iodine resistance, doctors use $5 \mathrm{mCi}$ instead of $30 \mathrm{mCi}$ for ${ }^{131}$ I when diagnosing whether thyroid tissue remains. Due to the interference of noise caused by SPECT's own imaging characteristics, equipment environment, and patient's condition, there are defects such as insignificant specificity of manual observation pictures, large basic dosage of ${ }^{131} \mathrm{I}$, long diagnosis time, low accuracy, and inconsistent standards, which lead to insufficient treatment pertinence, greatly increasing the blindness of treatment and the suffering of patients.

In the field of computer-aided diagnosis, the classification and prediction of thyroid tissue residues after ablation is still at a blank stage, but previous studies have confirmed that computeraided diagnosis technology based on medical images is an efficient method to improve diagnosis accuracy. Therefore, the goal of this paper is to design a computer-aided diagnosis system based on the deep convolutional neural network (DCNN) (8) to improve the accuracy of classification of thyroid tissue residues, aiming to verify the clinical role of the CAD system in the diagnosis of thyroid tissue residues.

\section{MATERIALS AND METHODS}

\subsection{Database}

All experimental protocols in this study were approved by the Second Affiliated Hospital of Guangxi University of Science and Technology. We collected a total of 446 SPECT images of thyroid cancer patients who received a low-dose ${ }^{131} \mathrm{I}$ diagnosis after total thyroidectomy. Among them, there were 346 SPECT images with residual thyroid tissue in the body and 100 SPECT images with no residual thyroid tissue in the body. The final diagnosis was given by two nuclear medicine doctors with more than 10 years of clinical diagnosis experience. In addition, all patients who were diagnosed with residual thyroid tissue in the body will receive a treatment dose of ${ }^{131} \mathrm{I}$ treatment, and the residual thyroid tissue can be clearly observed under treatment ${ }^{131} \mathrm{I}$ SPECT imaging. All patients who were diagnosed as having no residual thyroid tissue in the body will undergo a blood test for thyroid function, and all indicators are normal. Therefore, the accuracy of the label is guaranteed.

We refer to the ATA guidelines for the classification of high-, medium-, and low-risk recurrence populations and use the pathological results of the study subjects as the gold standard to count the number of high-, medium-, and low-risk recurrence populations in this study. Among them, 283 (63.4\%) patients were at a moderate risk of recurrence, 139 (31.2\%) patients were at a low risk of recurrence, and 24 (5.4\%) patients were at a high risk of recurrence. Generally speaking, people with low-risk recurrence risk are less likely to have cancer recurrence. People with high-risk recurrence risk can judge whether thyroid tissue remains from the level of thyroglobulin. In order to improve the generalization ability and applicability of the model, we adopted all the images as experimental objects. The plane images of the neck collected in this study were taken by Optima NM/CT 640 machine (GE Healthcare). The image acquisition protocol uses a high-energy, parallel-hole collimator, with an energy peak of $364 \mathrm{keV}$, a window width of $20 \%$, a matrix of $256 \times 256,5 \sim 10$ minutes/frame, and an imaging time of about $1 \mathrm{~h}$; the planar ${ }^{131} \mathrm{I}$ neck imaging was 
completed in both anterior and posterior projections. Table 1 shows the characteristics of the research objects.

\subsection{Image Processing}

The sensitivity of the SPECT image itself is relatively low and is affected by attenuation and scattering. There are background ghosts and edge artifacts in the image, which affect the accurate judgment of the disease. The main purpose of SPECT image processing is to remove noise and edge environment interference through digital image processing technology to accurately segment the thyroid bed area from the image. This paper uses python as the programming language and processes images based on the OpenCV open-source library.

\subsubsection{Histogram Equalization}

The images we collected are all standard medical images in DICOM format. In order to facilitate preprocessing and network training, we first convert them to JPG format images, centering on the thyroid bed area, and crop all image sizes to $800 \times 750$ size; this step is to remove irrelevant parts and information artifacts in the image. Figure 1 shows the original SPECT image without residue (A) and the original SPECT image with residue (B).

Histogram equalization is a commonly used gray-scale enhancement algorithm (9). It converts an image into an

TABLE 1 | Characteristics of study subjects.

\begin{tabular}{lc}
\hline Parameter & Value \\
\hline Mean age (years) & $41.8 \pm 11.4$ \\
Range & $13 \sim 79$ \\
Patient gender, $\mathbf{n}(\%)$ & $87(19.5 \%)$ \\
Male & \\
Female & $359(80.5 \%)$ \\
Pathology, $\mathbf{n}$ (\%) & $438(98.2 \%)$ \\
Papillary thyroid carcinoma & \\
Follicular thyroid carcinoma & $5(1.1 \%)$ \\
Medullary thyroid carcinoma & $0(0 \%)$ \\
Undifferentiated thyroid carcinoma & $3(0.7 \%)$ \\
\hline
\end{tabular}

\subsubsection{GrabCut}

In this paper, the GrabCut algorithm is used to segment the human thyroid bed area from the image to reduce the interference caused by background noise $(11,12)$. GrabCut is a man-machine interactive image segmentation method. It uses the texture color information and boundary contrast information in the image, and only a small amount of user interaction can get a better segmentation result. GrabCut uses the RGB three-channel image as the input image and takes the RGB three-channel Gaussian mixture model GMM as the target and

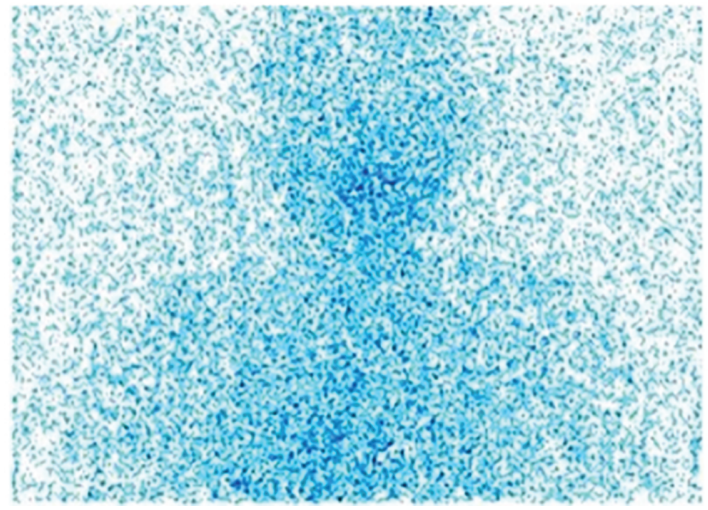

A

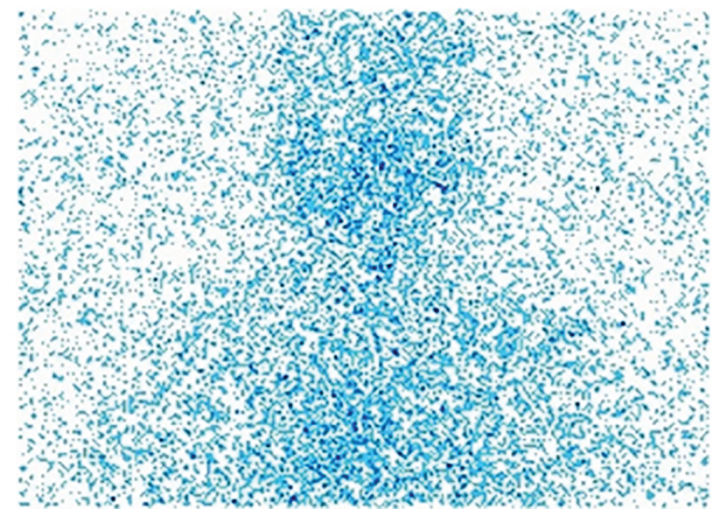

B

FIGURE 1 | (A) the original SPECT image with residue; (B) the original SPECT image without residue. 


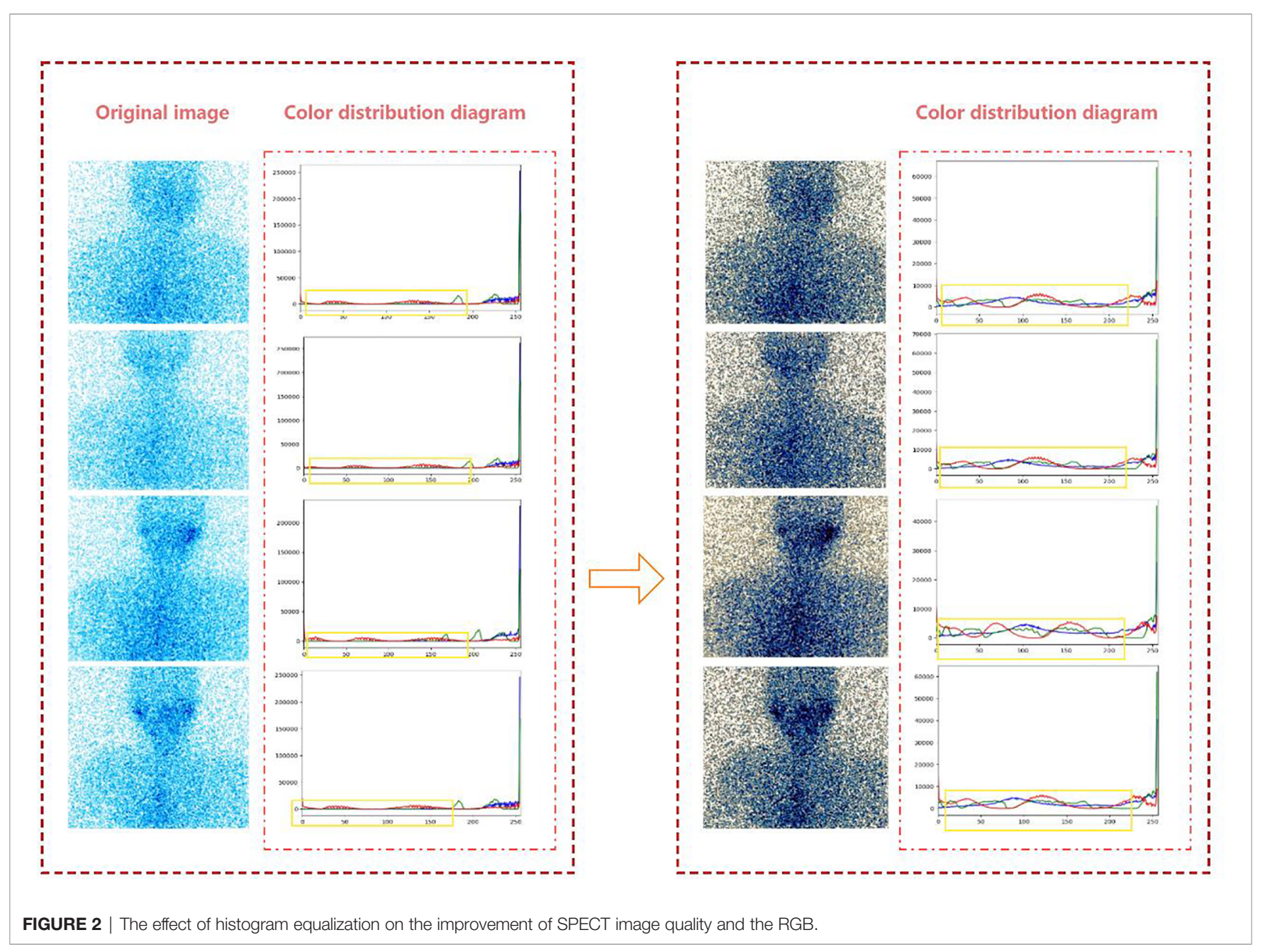

background model. Through continuous segmentation estimation and model parameter learning interactive iterations, the energy is minimized. The algorithm flow is mainly divided into two steps. The first step is initialization. Use a box to select the target in the input image. All the pixels outside the box are initialized to 0 as background pixels, and all the pixels in the box are initialized to 1 as possible target pixels. Then, the Gaussian mixture model of the target and background is estimated by pixel values. The target and background pixels are clustered into $\mathrm{K}$ classes through K-means clustering to obtain K Gaussian models. Each Gaussian mixture model calculates the mean and covariance of each Gaussian component through pixel samples. The second step is iterative minimization. The pixel value is substituted into the Gaussian component in the Gaussian mixture model of the result of the first step, the probability of each component is compared, and the pixel is assigned to the Gaussian component with the highest probability according to the result. Figure 3 shows the calculation process of the GrabCut algorithm.

Repeat the above two steps. In the iterative process, each pixel belongs to the background pixel or the target pixel will continue to change, and the energy function will continue to decrease to ensure that it can eventually converge (13). Figure 4 shows the segmentation effect of GrabCut on the SPECT image.

\subsection{Deep Residual Learning ResNet-18 Model}

The deep residual learning network was developed by researchers from Microsoft Research, and they won the first place in all the tracks in the ImageNet and COCO $(14,15) 2015$ competitions. The difficulty of training deep networks is related to the back propagation of gradients. The deeper the network layer, the more difficult it is to update the gradient of the lower layer (the first layer). Therefore, the deep architecture cannot actually update these layers. ResNet developers think of the concept of residual representation commonly used in the computer field and apply it to the construction of ordinary CNN models. On the basis of ordinary networks, they form a new connection by adding a jump connection between each two layers of networks. The module is called the residual block. The author proves through experiments that ResNet can easily surpass the depth of traditional CNN through residual blocks and has a faster learning convergence speed and a more significant classification effect. The basic structure of the residual block is shown in Figure 5. 

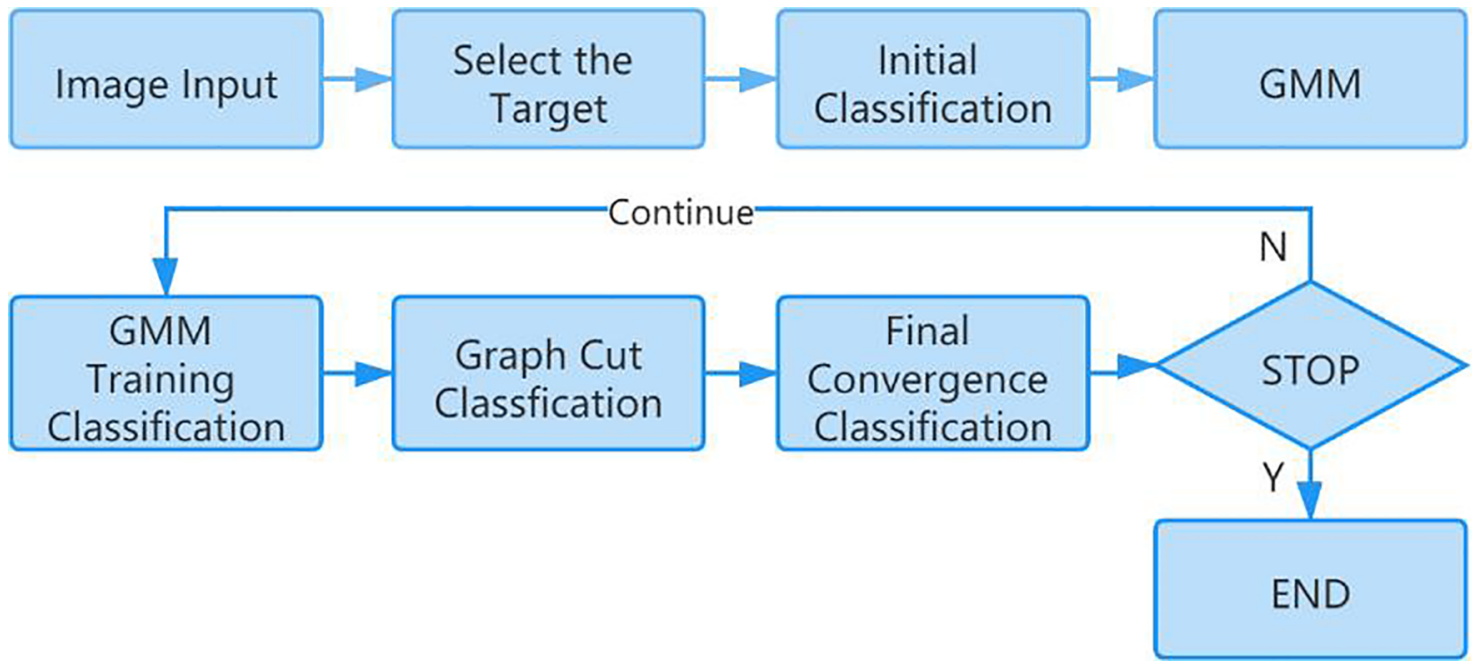

FIGURE 3 | The calculation process of the GrabCut algorithm.
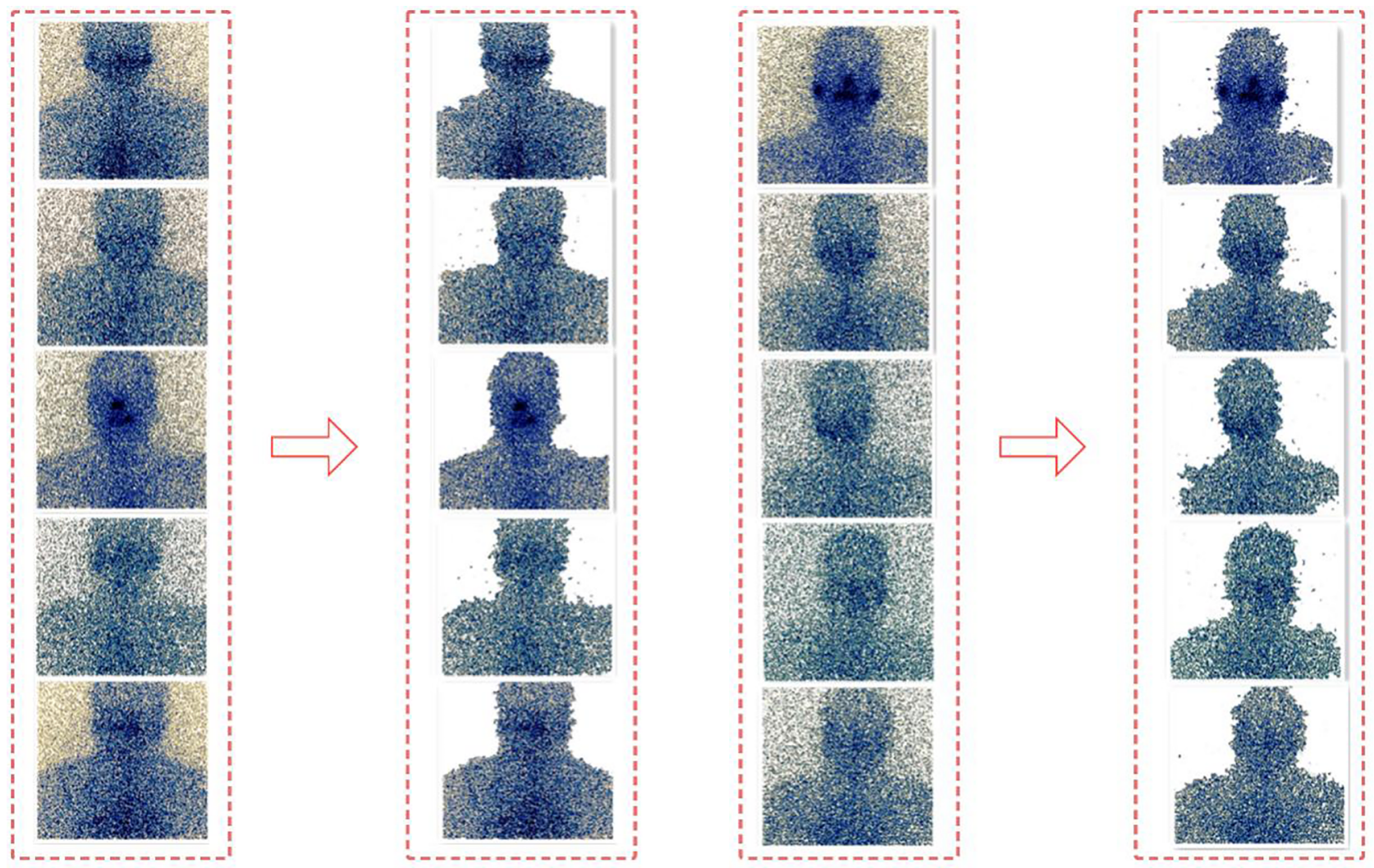

FIGURE 4 | The segmentation effect of Grabcut on the SPECT.

ResNet-18 (16) has eight residual blocks; each block includes two convolutional layers, batch normalization layer and ReLU activation function. The output of each block is merged with its own input. Each convolutional layer in the residual block uses a
$3 \times 3$ convolution kernel. In the first layer of the network, ResNet uses a $7 \times 7$ convolution with a step size of 2 to downsample the input and pooling layers. The last layer is the average pooling layer, which creates 1,000 feature maps based on ImageNet data. 


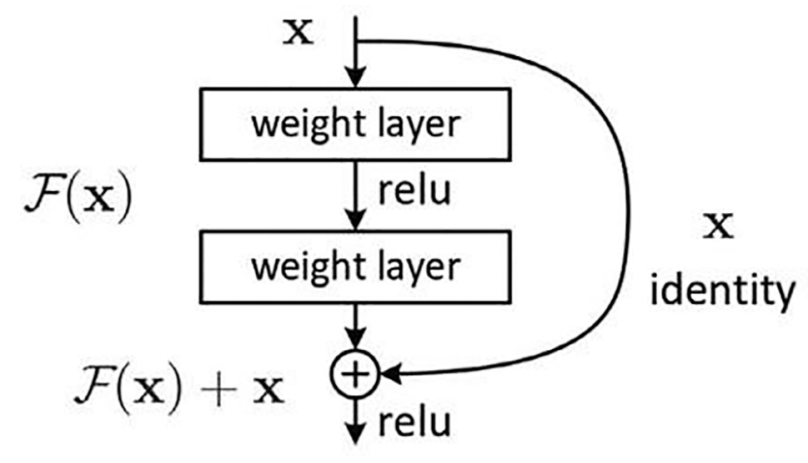

FIGURE 5 | The basic structure of the Resnet.

The result will be a 1,000-dimensional vector, which is then directly sent to the Softmax layer for classification. The network architecture of ResNet-18 is shown in Figure 6.

Due to the particularity of SPECT images, it is difficult for us to obtain large training samples as support to train convolutional neural network models from scratch, and it is very difficult to train deep learning models on a small amount of data. Therefore, we use a pretrained convolutional neural network to fine-tune the method to deal with this problem. In the classification task, the convolution part of the deep-learning model usually serves as the task of feature extraction, which can be transferred to related but different classification tasks by means of transfer learning. Although ultrasound images are very different from natural images, their perception of features is the same. The more samples you train, the more common the features will be. Fine-tuning is the process of adapting the weights of pretrained $\mathrm{CNN}$ to different data sets by using backpropagation. Fine-tuning a pretrained network is usually much faster and easier than training a $\mathrm{CNN}$ or neural network from scratch (17-19). We first pretrain ResNet-18 on the ImageNet dataset containing 1,000 types of natural images, so that it can learn various common image features and obtain a set of powerful parameters for initialization. Subsequently, we transfer the learned features and parameters from the general domain to the classification problem of SPECT images. The last fully connected layer of the original ResNet-18 outputs 1,000 categories of the ImageNet dataset. We fine-tune ResNet-18 by initializing a new fully connected layer, training on new SPECT image data, and learning and updating the parameters and weights of the fully connected layer through the backpropagation algorithm, so that the model only outputs two classes. That is, there is or no thyroid tissue remaining in the body.

Deep learning is developing rapidly in the field of computer vision; sophisticated and excellent advanced models have sprung up like mushrooms. They have achieved amazing accuracy in all major lists. However, limited by the actual application scenarios and resource equipment of the hospital, it is difficult to show the power of these complex models. Therefore, in real scenarios, lightweight models are more popular with people, and the actual application range is wider. Therefore, we decided to use the same fine-tuning technique to compare the proposed network architecture with the widely used lightweight networks SqueezeNet $(20,21)$ and ShuffleNetV2 $(22,23)$. Table 2 shows the network architecture of ShuffleNetv2, and Figure 7 shows the network architecture of SqueezeNet.

\subsection{Model Evaluation Index}

In this paper, classification accuracy, sensitivity (recall rate), specificity, precision, F1-Score, and area under the receiver operating characteristic curve (AUC) are used to evaluate the performance of the two classification models. The accuracy rate represents the correct diagnosis rate of whether thyroid tissue remains. Sensitivity refers to the percentage of non-residue-type SPECT images correctly diagnosed as non-residue. Specificity refers to the percentage of SPECT images with residual types that are correctly classified as having residuals. The precision rate refers to the ratio of images that are predicted to have residual images that are actually residual images. The F1-Score indicator

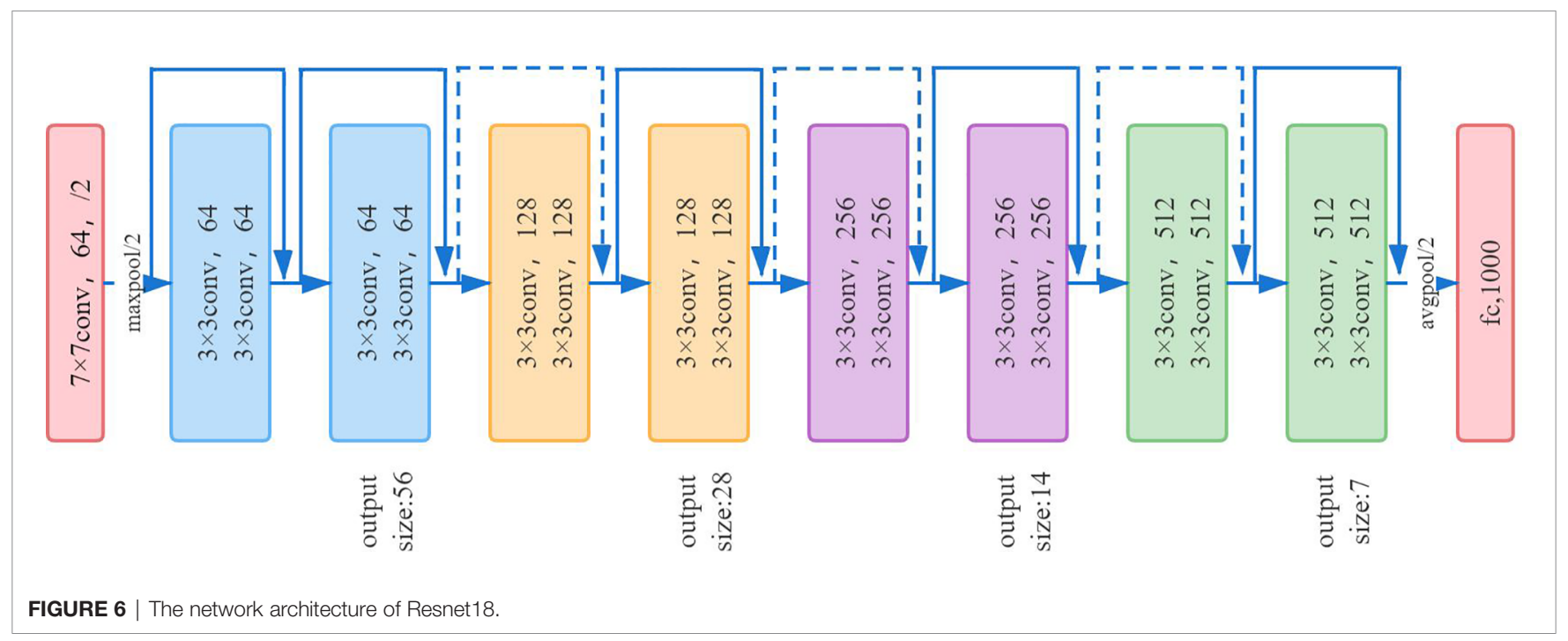


TABLE 2 | Overall architecture of ShuffleNetv2.

\begin{tabular}{|c|c|c|c|c|c|c|c|c|}
\hline \multirow[t]{2}{*}{ Layer } & \multirow[t]{2}{*}{ Output size } & \multirow[t]{2}{*}{ Ksize } & \multirow[t]{2}{*}{ Stride } & \multirow[t]{2}{*}{ Repeat } & \multicolumn{4}{|c|}{ Output channels } \\
\hline & & & & & $0.5 x$ & $1 x$ & $1.5 x$ & $2.5 x$ \\
\hline Image & $224 \times 224$ & & & & 3 & 3 & 3 & 3 \\
\hline \multirow[t]{2}{*}{ Conv1 MaxPool } & $112 \times 112$ & $3 \times 3$ & 2 & 1 & 24 & 24 & 24 & 24 \\
\hline & $56 \times 56$ & $3 \times 3$ & 2 & & & & & \\
\hline \multirow[t]{2}{*}{ Stage2 } & $28 \times 28$ & & 2 & 1 & 48 & 116 & 176 & 244 \\
\hline & $28 \times 28$ & & 1 & 3 & & & & \\
\hline \multirow[t]{2}{*}{ Stage3 } & $14 \times 14$ & & 2 & 1 & 96 & 232 & 352 & 488 \\
\hline & $14 \times 14$ & & 1 & 7 & & & & \\
\hline \multirow[t]{2}{*}{ Stage4 } & $7 \times 7$ & & 2 & 1 & 192 & 464 & 704 & 976 \\
\hline & $7 \times 7$ & & 1 & 3 & & & & \\
\hline Conv5 & $7 \times 7$ & $1 \times 1$ & 1 & 1 & 1024 & 1024 & 1024 & 2048 \\
\hline GlobalPool & $1 \times 1$ & $7 \times 7$ & & & & & & \\
\hline FC & & & & & 1,000 & 1,000 & 1,000 & 1,000 \\
\hline
\end{tabular}

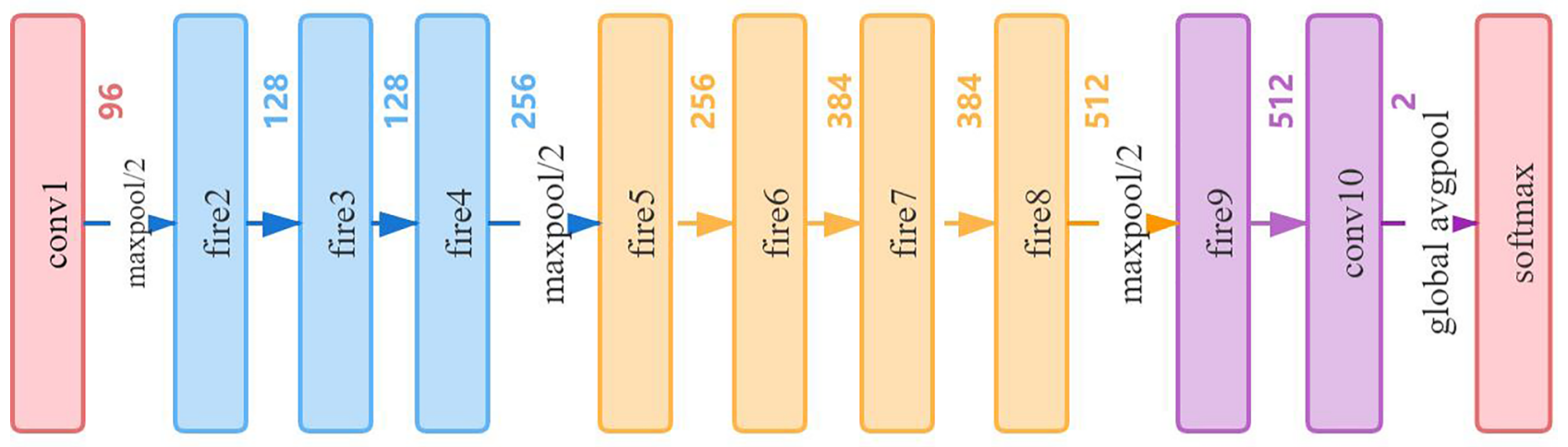

FIGURE 7 | The network architecture of SqueezeNet.

combines the results of precision and recall. The value range of F1-Score is from 0 to 1 . 1 represents the best output of the model, and 0 represents the worst output of the model. The ROC curve is a tool to measure the balance between finding true positives and avoiding false positives. The AUC value is within the interval of $[0.5,1]$, indicating the possibility of correctly predicting whether the thyroid tissue remains in the body. A good classifier has an AUC close to 1.

\section{RESULTS}

\subsection{Dataset Division}

Due to the small data set, in the case of insufficient sample size, in order to make full use of the data set to test the effect of the model, this paper takes a random grouping experiment $(24,25)$; a total of 10 experiments have been carried out. In each randomized experiment, the various images in the data set are randomly divided at a ratio of 3:1. The training set contains 335 images, including 260 images of residual type and 75 images of non-residual type. The test set contains 111 images, of which 86 are residual types and 25 are non-residual types. The data division is shown in Table 3.

\subsection{Fine-Tuning ResNet-18}

The pretrained ResNet-18 model has been trained on the ImageNet dataset, and it contains the weights and biases that represent the features of the ImageNet dataset. Therefore, the pretrained model has learned common features including curves and edges, and these features can also be used in our classification problems. In this part of the work, we transfer the knowledge obtained from the ImageNet dataset from the pretrained model to another model and modify the final fully connected layer of the pretrained ResNet-18 to adapt ResNet-18 to our SPECT dataset. Figure 8 shows the fine-tuning process of the model.

In order to obtain the best learning performance, it is very important to set appropriate parameter values of the backpropagation algorithm. Learning rate, momentum rate, and activation function are the main parameters that affect the learning

TABLE 3 | Number of images in the training set and test set.

\begin{tabular}{lccc}
\hline & Total & Residual & Non-residual \\
\hline Training set & 335 & 260 & 75 \\
Testing set & 111 & 86 & 25
\end{tabular}




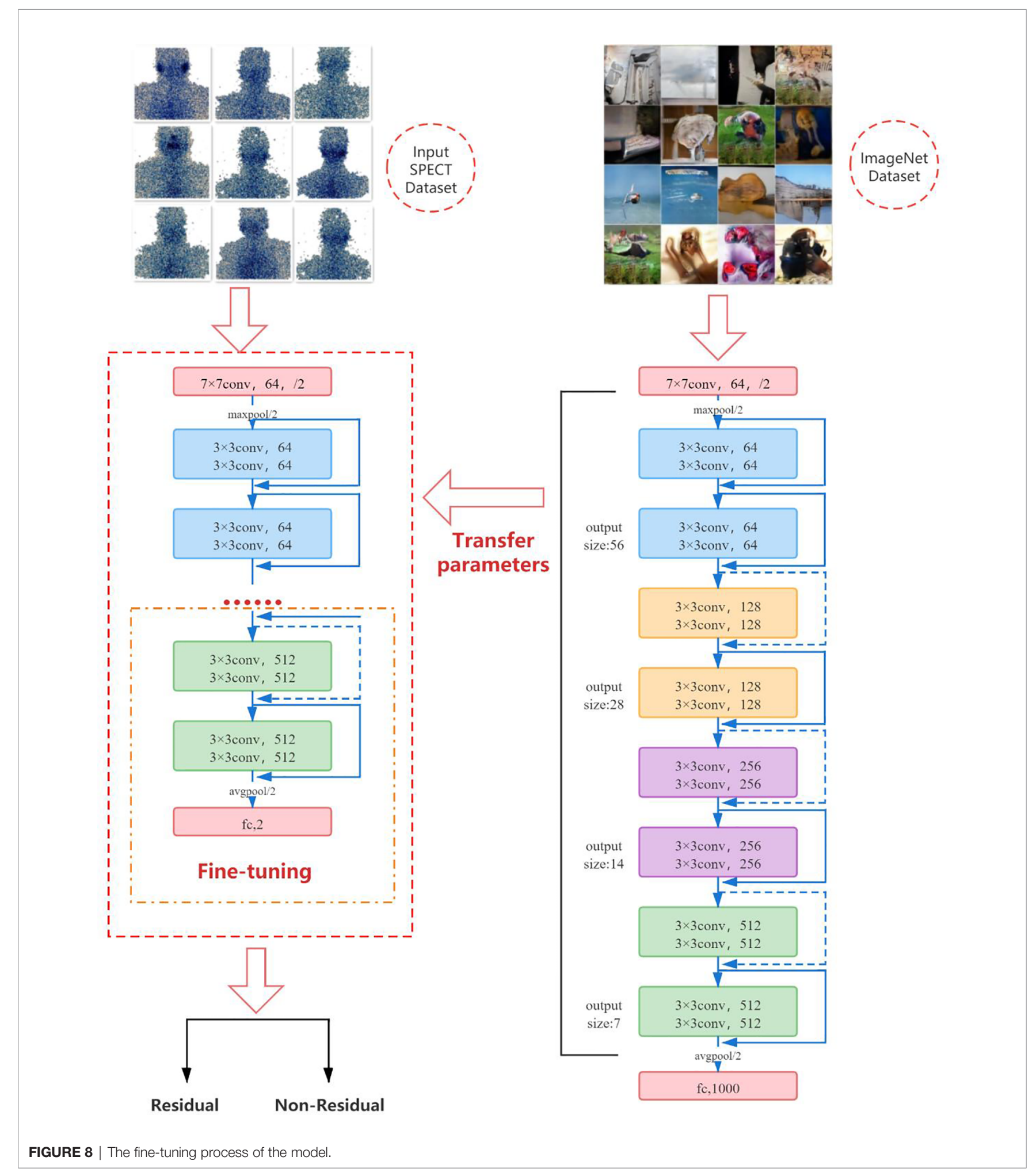

performance of DCNN in the backpropagation algorithm. However, there are no clear guidelines for the selection of the best values of these parameters. The only way to check which parameters perform better is through multiple trials and errors. A higher learning rate value often leads to overfitting, while a lower learning rate value leads to limited error changes in different periods. In our experiments, the learning rate is chosen to be $10^{-5}$ through the experience of controlling the verification error in the fine-tuning process, so as to smoothly adapt to the new task without severely destroying the acquired knowledge. In the process of 
training and verification, the optimization algorithm Adam (26), which is an adaptive learning rate, is selected for parameter optimization. Compared with other optimization functions, Adam can calculate different adaptive learning rates for different parameters, and the memory requirements are relatively small. Moreover, the weight attenuation coefficient is $10^{-5}$. When the classifier output layer processes two classes, Softmax is the most appropriate as the activation function.

Before putting the image data into network training, we also performed some processing on the data set. The first is to deal with the problem of class imbalance. We use the Weighted RandomSampler sampler under the PyTorch framework to perform weight sampling and use the idea of sampling from a set of samples based on polynomial distribution to assign weights to samples of different categories. Finally, a corresponding weight is added to each sample, and the number of images of different categories can be balanced during sampling. Then, each image put into training is sized to $224 \times 224$ for ResNet-18 training, and finally the image channels are standardized with the aim to prevent the convergence of too large numerical effects of the results. Standardization can accelerate the speed of convergence and to a certain extent can also improve the accuracy of convergence. We tried to increase the number of epochs from 30. Starting from 50 epochs, the accuracy of the model has not been greatly improved. When the epoch is set to 80 , the model performance is the most stable and the loss curve is the most stable. The changes in accuracy and loss during the training process are shown in Figure 9.

\subsection{Comparison of Diagnosis Performance of Different CAD Systems}

All experiments use the same hardware configuration. The model used in this article has the advantage of a small amount of training parameters. The experiment can be performed on a personal computer device. All experiments are performed on the computer configured with Intel Core i7-7700 3.60 GHz CPU, 8 GB memory, and Windows 10 as the operating system. To assess the statistical significance of the performance differences, the

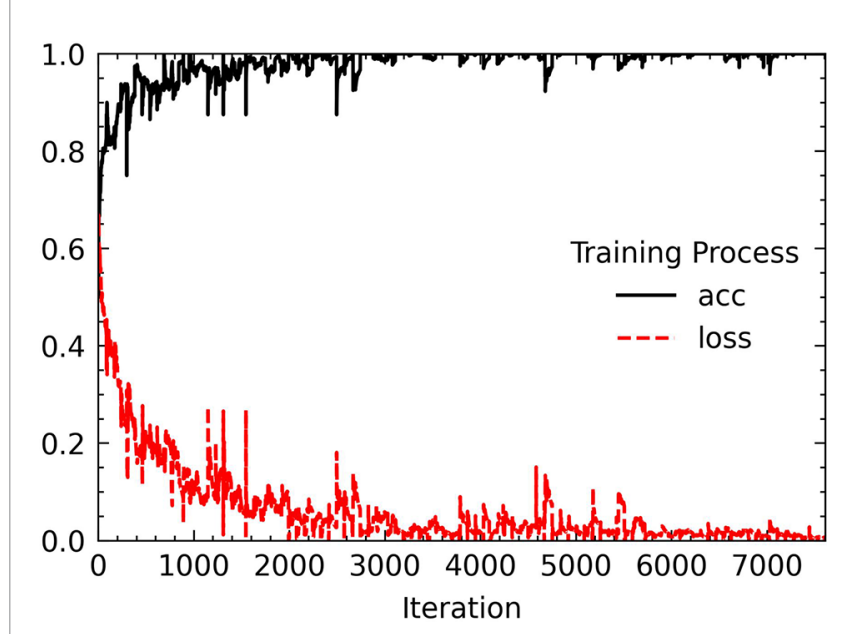

FIGURE 9 | The training process of the proposed model. non-parametric Mann-Whitney $\mathrm{U}$ test was performed for the values of each evaluation index. Areas under the ROC curve between each model were compared using the method of DeLong et al.

Table 4 summarizes the performance of the proposed model in this article and the two lightweight models that are widely used in the diagnosis of residual thyroid tissue. Specifically, the classification accuracy of the proposed model in this paper is $96.69 \% \pm 1.27 \%$, the sensitivity is $94.75 \% \pm 1.53 \%$, the specificity is $99.6 \% \pm 1.26 \%$, the precision is $99.96 \% \pm 0.09 \%$, and the F1-Score is $97.55 \% \pm 0.90 \%$. Comparing the proposed model with the other two models, respectively, the accuracy and sensitivity of the proposed model and the other two models are statistically significant $(\mathrm{p}<0.05)$, indicating that the proposed model has a better ability to diagnose residual thyroid tissue. There is no significant difference between the proposed model and the other two models in terms of specificity and sensitivity $(p>0.05)$. Figure 10 shows the ROC curve of the proposed model and two lightweight models when judging whether thyroid tissue remains. The area under the curve values of the proposed model, SqueezeNet, and ShuffleNetv2 are 0.988 (95\% CI, 0.941-1.000), 0.898 (95\% CI, $0.819-0.951)(\mathrm{p}=0.0257)$, and 0.885 (95\% CI, 0.803-0.941) $(\mathrm{p}=$ 0.0057 ), indicating that the accuracy of the proposed model is higher than that of SqueezeNet and ShuffleNetv2. These results mean that almost all SPECT images can be correctly classified by ResNet-18. At the same time, it is proved that fine-tuning the existing DCNN to generate a deep learning network in a specific field can effectively extract the high-level features of the SPECT image of the thyroid bed area and perform high-precision classification of whether there is residual thyroid tissue in the body after total thyroidectomy of thyroid cancer patients.

\section{DISCUSSION}

Computer-aided diagnosis (CAD) is a computerized technology that can be used as a second objective and accurate opinion. It can improve the accuracy and consistency of radiological diagnosis, reduce the reading time of radiological examination, and help radiologists perform interpretation and diagnosis of medical images (27). In recent years, computer-aided diagnosis (CAD) based on medical images, as a method that can effectively eliminate operation dependence and improve the accuracy of diagnosis, has performed very well in many disease diagnosis studies. CAD usually consists of three main steps: preprocessing, feature extraction, and classification. Generally speaking, preprocessing includes image denoising, contrast enhancement, edge enhancement, and segmentation. These steps not only are cumbersome but also have a great impact on subsequent processing. Extracting image features can be divided into morphological features and texture features extracted by machine learning models or using convolutional neural networks to extract feature maps. The classifier can be a support vector machine (SVM), k-nearest neighbor, AdaBoost, Gaussian mixture model, probabilistic neural network, decision tree, random forest, or Softmax. For example, Zhang et al. (28) established a CAD model on the ultrasound data set of 303 
TABLE 4 | Performance of each model in 10 randomized grouping experiments.

\begin{tabular}{|c|c|c|c|c|c|}
\hline & Proposed model & SqueezeNet & $p$ value & ShuffleNetv2 & $p$ value \\
\hline ACC (\%) & $96.69(1.27)$ & $90.26(0.98)$ & $1.107 e^{-5}$ & $86.39(1.47)$ & $1.111 e^{-5}$ \\
\hline SEN (\%) & $94.75(1.53)$ & 88.66 (1.89) & $1.111 e^{-5}$ & 81.7 (1.99) & $1.119 e^{-5}$ \\
\hline SPE (\%) & $99.6(1.26)$ & $98.8(1.93)$ & 0.168 & $98.4(2.80)$ & 0.297 \\
\hline PPV (\%) & $99.96(0.09)$ & $99.89(0.17)$ & 0.148 & $99.85(0.24)$ & 0.246 \\
\hline F1 score (\%) & $97.55(0.90)$ & $92.73(0.75)$ & $4.553 e^{-6}$ & $89.18(0.70)$ & $1.021 e^{-5}$ \\
\hline
\end{tabular}

Numbers format: mean value (standard deviation).

patients with thyroid cancer to classify and predict different types of malignant nodules. The specificity reached $86 \%$ and was no different from that of a senior radiologist. The sensitivity and accuracy of $78.6 \%$ and $90.7 \%$ are lower than those of senior radiologists, but they can significantly improve the diagnostic level of inexperienced radiologists. Ali et al. (29) established a deep artificial neural network model for lung cancer prediction based on 888 CT images of lung nodules from the LIDC/IDRI database. The accuracy was $99.1 \%$, the sensitivity was $99.2 \%$, and the specificity was $99.1 \%$. Peng et al. (30) developed a deep learning artificial intelligence model (ThyNet) on thyroid nodule ultrasound images of 8,339 patients to distinguish malignant and benign thyroid nodules, achieving a significantly higher AUC (0.922 vs. 0.839) than radiologists and effectively reducing the number of unnecessary fine needle punctures in a simulated scenario. Fujisawa et al. (31) established a deep convolutional neural network model (DCNN) on the clinical image data set of 1,842 skin tumor patients from the University of Tsukuba Hospital to classify and diagnose 14 diseases. DCNN's classification of skin tumor images reached $92.4 \%$. The accuracy rate is higher than that of the committee-certified dermatologist (85.3\%). Shakarami et al.

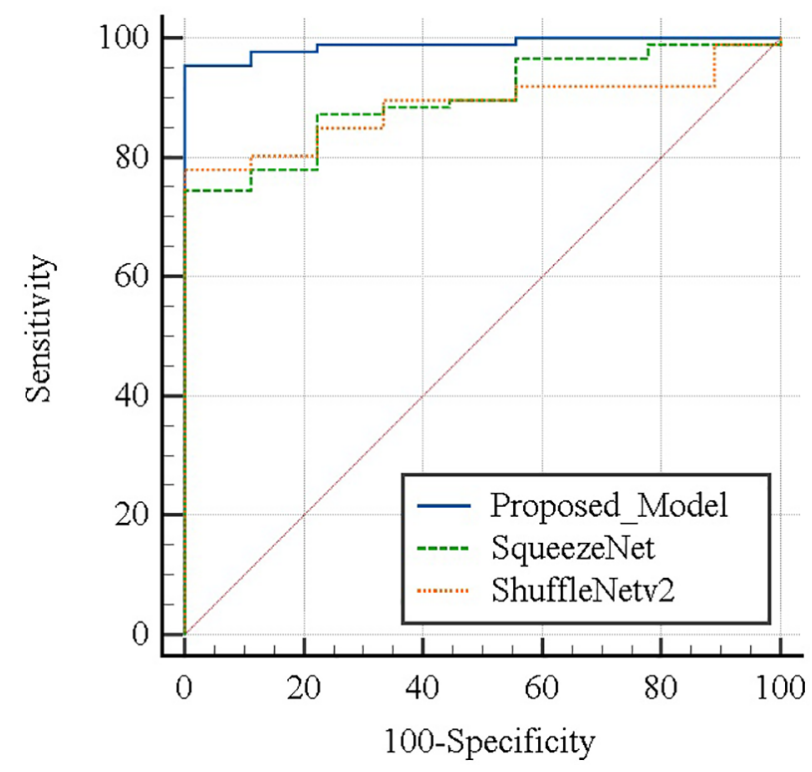

FIGURE 10 | Areas under the ROC curve between each model were compared using the method of DeLong et al.
(32) proposed a computer-aided diagnosis system COV-CAD to diagnose COVID-19 disease through lung images. The system uses fine-tuned AlexNet-CNN to extract features and uses a majority voting method to integrate multiple classifiers for final diagnosis; the accuracy rates of CT and X-ray data sets are $93.20 \%$ and $99.38 \%$, respectively. Deepak et al. (33) studied the classification of brain tumors. Using the concept of deep transfer learning, pretrained GoogLeNet is used to extract features from brain MRI images, and a validated classifier model is integrated to classify the extracted features to distinguish gliomas, meningiomas, and pituitary tumors. A 98\% classification accuracy rate was achieved on the MRI data set from figshare. Liu et al. (34) established a computeraided diagnosis system for thyroid diseases based on the SPECT image data set to classify and predict hyperthyroidism, hypothyroidism, and normal and evaluated the classification effects of four CNN models. Among them, the VGG16 model has the best performance, with an accuracy rate of $96.2 \%$ and an AUC of 99.6\%. Ma et al. (35) proposed a computer-aided diagnosis method based on the convolutional neural network (CNN) in 2018 and developed an improved $\mathrm{CNN}$ method with enhanced structure for deep learning to improve the performance of CNN. The proposed CNN method obtains the best accuracy and smaller error in the confusion matrix, can maintain performance under different iteration times, and has superior performance in the diagnosis of thyroid diseases. Furthermore, the DenseNet network was used to establish the diagnosis model of thyroid disease based on SPECT images in 2019, and its structure and training methods were improved on the basis of the traditional DenseNet network structure, which greatly improves the diagnosis effect of Graves' disease, Hashimoto disease, and subacute thyroiditis (36).

In recent years, treatment strategies for thyroid cancer all over the world have the defect of overtreatment. In the diagnosis process of the doctor based on the SPECT image to determine whether there is thyroid tissue remaining in the body of a patient with thyroid cancer after ablation, in order to ensure that the suspected remaining patient is not at risk of recurrence, a certain dose of ${ }^{131}$ I will be given to the patient to remove these possible remaining thyroid tissue. Therefore, it is found that while doctors are making long-term plans for the patient's physical condition, it is also difficult to avoid the occurrence of overtreatment problems. In this regard, we can pay attention to the specificity of the model, and we can find that the three models can reach more than $98 \%$ at the same time, which means that patients with no residual thyroid tissue in the body have a $98 \%$ chance of being correctly diagnosed. In addition, the precision rate is also an indicator worth noting. The precision of the three models is greater than $99 \%$, which means that all patients with residuals predicted by the model are real patients 
with residuals. In other words, it represents that the probability of a patient with no residues in the body being misjudged as having residues is close to 0 . Doctors can refer to the results of such classifications to reduce the dose of ${ }^{131} \mathrm{I}$ medication for such patients, so as to reduce the harm to the patient's body and reasonably reduce the occurrence of excessive treatment. On the other hand, we must also be able to identify as many patients as possible with real residual thyroid tissue in the body. In terms of sensitivity, the ResNet-18 model is significantly better than SqueezeNet and ShuffleNetv2. It can identify patients with residual thyroid tissue in the body with $96.69 \%$ accuracy, which shows that it can provide doctors with objectives and valuable second opinions and help them clarify the residual condition of thyroid tissue, reduce the workload, and avoid misdiagnosis due to visual fatigue.

Compared with the diagnosis research of some other diseases in the field of computer-aided diagnosis (19), the network model we proposed has the advantages of being light weight, having fewer training parameters, and having shorter training times while ensuring performance. It has the advantages of more convenience in experiments and applications. When our proposed method can be evaluated on a larger data set, it can be used as an auxiliary diagnostic tool in daily clinical practice without incurring additional installation costs. In addition, it can also save clinical diagnosis time. The model proposed in this paper takes an average of $20 \mathrm{~s}$ to make a diagnosis of an image. According to the nuclear medicine doctor of The Second Affiliated Hospital of Guangxi University of Science and Technology, it takes about $10 \mathrm{~min}$ to make a clinical diagnosis of an image. Therefore, we conservatively estimate that on each image, it can save clinicians at least $5 \mathrm{~min}$ on average. We recognize that this is an exploratory study, and our data set cannot adequately cover or represent the actual population base in clinical practice. Currently, we do not have enough data sets to verify the generalization ability of the model. With the continuous increase of clinical data, in the future work, we will more finely classify the residual thyroid tissue of patients with thyroid cancer after thyroid ablation, will predict the general situation of the residual thyroid tissue in the patient's body, and can give more reference suggestions when the doctor chooses a more appropriate dose for the patient.

\section{CONCLUSION}

This paper is an exploratory work that applies deep learning to the classification of thyroid tissue residues in patients with thyroid cancer after ablation. We studied the feasibility of finetuning the deep convolutional neural network based on SPECT images to classify and predict the residual thyroid tissue in patients with thyroid cancer after ablation. We first use histogram equalization and GrabCut method to preprocess the SPECT image and propose a method of fine-tuning ResNet-18, which is applied to our data set through transfer learning. Finally, experiments were conducted on SPECT images of 446 patients and performance comparisons were made with the widely used lightweight network SqueezeNet and ShuffleNetV2 models. The accuracy, sensitivity, specificity, precision and F1-Score of the proposed model are $96.69 \%, 94.75 \%, 99.6 \%$, 99.96\%, and $97.55 \%$, respectively, and the AUC is 0.988. Experiments have proved that the strategy of fine-tuning the pretraining DCNN model in this paper and learning the relevant features of image classification on the natural image data set is beneficial to improving the training process and can successfully transfer the knowledge and parameters obtained by pretraining to the task of thyroid tissue residual classification, The thyroid tissue residue classification system based on the deep residual network ResNet-18 proposed in this paper can be used as a computeraided diagnosis method to improve the diagnosis of thyroid tissue residues in patients with thyroid cancer after ablation. While more accurately diagnosing patients with residual thyroid tissue in the body, we have tried our best to avoid the occurrence of overtreatment.

\section{DATA AVAILABILITY STATEMENT}

The raw data supporting the conclusions of this article will be made available by the authors, without undue reservation.

\section{ETHICS STATEMENT}

The studies involving human participants were reviewed and approved by the Medical Ethics Committee of the Second Affiliated Hospital of Guangxi University of Science and Technology. Written informed consent for participation was not required for this study in accordance with the national legislation and the institutional requirements.

\section{AUTHOR CONTRIBUTIONS}

YG and JX were major contributors in writing the manuscript, have contributed equally to this work, and share first authorship. YG, JX, XL, XY, and WP conceived and designed the experiments. $\mathrm{SM}, \mathrm{DH}$, and MQ organized the database. YG and LZ did the literature research. All authors contributed to the manuscript revision and read and approved the submitted version.

\section{FUNDING}

This work was supported by grants from the Young and Middleaged Teachers' Scientific Research Basic Ability Promotion Project of Guangxi Universities (2021KY0339), Doctoral program of Guangxi University of Science and Technology (21Z01 and 21Z24), and Self-funded Scientific Research Project of Guangxi Health Commission (Z20212280).

\section{ACKNOWLEDGMENTS}

We would like to thank Zhenbing Liu of the Department of Computer and Information Security of Guilin University of Electronic Technology for his excellent suggestions on model selection and improvement. 


\section{REFERENCES}

1. Xue YL, Qiu ZL, Song HJ, Luo QY. Value of I-131 Spect/Ct for the Evaluation of Differentiated Thyroid Cancer: A Systematic Review of the Literature. Eur J Nucl Med Mol Imaging (2013) 40:768-78. doi: 10.1007/s00259-012-2310-x

2. Wang J, Li S. A Brief Report on the Results of the National Survey of Nuclear Medicine in 2020. Chin J Nucl Med Mol Imaging Chin J Nucl Med Mol Imaging (2020) 40:747-9. doi: 10.3760/cma.j.cn321828-20201109-00403

3. Schmidt D, Szikszai A, Linke R, Bautz W, Kuwert T. Impact of 131i Spect/ Spiral Ct on Nodal Staging of Differentiated Thyroid Carcinoma at the First Radioablation. J Nucl Med (2009) 50:18-23. doi: 10.2967/jnumed.108.052746

4. Jiang L, Xiang Y, Huang R, Tian R, Liu B. Clinical Applications of SinglePhoton Emission Computed Tomography/Computed Tomography in PostAblation 131iodine Scintigraphy in Children and Young Adults With Differentiated Thyroid Carcinoma. Pediatr Radiol (2021) 51:1724-31. doi: 10.1007/s00247-021-05039-2

5. Malamitsi JV, Koutsikos JT, Giourgouli SI, Zachaki SF, Pipikos TA, Vlachou FJ, et al. I-131 Postablation Spect/Ct Predicts Relapse of Papillary Thyroid Carcinoma More Accurately Than Whole Body Scan. Vivo (2019) 33:225563. doi: 10.21873/invivo.11731

6. Haugen BR, Alexander EK, Bible KC, Doherty G, Mandel SJ, Nikiforov YE, et al. American Thyroid Association Management Guidelines for Adult Patients With Thyroid Nodules and Differentiated Thyroid Cancer: The American Thyroid Association Guidelines Task Force on Thyroid Nodules and Differentiated Thyroid Cancer. Thyroid Off J Am Thyroid Assoc (2015) 2165:55-6. doi: 10.1089/thy.2015.0020

7. Kao CH, Yen TC. Stunning Effects After a Diagnostic Dose of Iodine-131. Nuklearmedizin Nucl Med (1998) 37:30-2. doi: 10.1055/s-0038-1629854

8. Moussa O, Khachnaoui H, Guetari R, Khlifa N. Thyroid Nodules Classification and Diagnosis in Ultrasound Images Using Fine-Tuning Deep Convolutional Neural Network. Int J Imaging Syst Technol (2020) 30:185-95. doi: 10.1002/ima.22363

9. Verdenet J, Cardot JC, Baud M, Chervet H, Duvernoy J, Bidet R. Scintigraphic Image Contrast-Enhancement Techniques: Global and Local Area Histogram Equalization. Eur J Nucl Med (1981) 6:261-4. doi: 10.1007/BF00251349

10. Kuo CFJ, Wu HC. Gaussian Probability Bi-Histogram Equalization for Enhancement of the Pathological Features in Medical Images. Int J Imaging Syst Technol (2019) 29:132-45. doi: 10.1002/ima.22307

11. Rother C, Kolmogorov V, Blake A. Grabcut - Interactive Foreground Extraction Using Iterated Graph Cuts. ACM SIGGRAPH 2004, SIGGRAPH 2004, August 8, 2004 - August 12, 2004 (Association for Computing Machinery). In: ACM SIGGRAPH 2004 Papers, SIGGRAPH 2004 New York, NY, USA: Association for Computing Machinery (2004). p. 309-14. doi: 10.1145/1186562.1015720

12. Lu YW, Jiang JG, Qi MB, Zhan S, Yang J. Segmentation Method for Medical Image Based on Improved Grabcut. Int J Imaging Syst Technol (2017) 27:38390. doi: 10.1002/ima.22242

13. Deng LL. Pre-Detection Technology of Clothing Image Segmentation Based on Grabcut Algorithm. Wirel Pers Commun (2018) 102:599-610. doi: $10.1007 /$ s11277-017-5050-1

14. Lin TY, Maire M, Belongie S, Hays J, Perona P, Ramanan D, et al. Microsoft COCO: Common Objects in Context. Zurich, Switzerland: Springer International Publishing (2014) p. 740-55. doi: 10.1007/978-3-319-10602-1_48

15. Luo S, Kim EH, Dighe M, Kim Y. Thyroid Nodule Classification Using Ultrasound Elastography via Linear Discriminant Analysis. Ultrasonics (2011) 51:425-31. doi: 10.1016/j.ultras.2010.11.008

16. He K, Zhang X, Ren S, Sun J. Deep Residual Learning for Image Recognition. 29th IEEE Conference on Computer Vision and Pattern Recognition, CVPR 2016, June 26, 2016 - July 1, 2016 (IEEE Computer Society) (2016). In: Proceedings of the IEEE Computer Society Conference on Computer Vision and Pattern Recognition. Las Vegas, NV, USA: IEEE (2016). p. 770-8. doi: 10.1109/ CVPR.2016.90

17. Chi JN, Walia E, Babyn P, Wang J, Groot G, Eramian M. Thyroid Nodule Classification in Ultrasound Images by Fine-Tuning Deep Convolutional Neural Network. J Digit Imaging (2017) 30:477-86. doi: 10.1007/s10278017-9997-y

18. Vasile CM, Udriștoiu AL, Ghenea AE, Popescu M, Gheonea C, Niculescu CE, et al. Intelligent Diagnosis of Thyroid Ultrasound Imaging Using an Ensemble of Deep Learning Methods. Medicina (2021) 57:395. doi: 10.3390/medicina57040395
19. Yu J, Deng Y, Liu T, Zhou J, Jia X, Xiao T, et al. Lymph Node Metastasis Prediction of Papillary Thyroid Carcinoma Based on Transfer Learning Radiomics. Nat Commun (2020) 11:4807. doi: 10.1038/s41467-020-18497-3

20. Forrest, Han S, Matthew, Ashraf K, William, Keutzer K. Squeezenet: AlexnetLevel Accuracy With 50x Fewer Parameters and !0.5mb Model Size. ArXiv Pre-Print Server (2016) 3:35-38.

21. Kim JE, Nam NE, Shim JS, Jung YH, Cho BH, Hwang JJ. Transfer Learning via Deep Neural Networks for Implant Fixture System Classification Using Periapical Radiographs. J Clin Med (2020) 9:11. doi: 10.3390/jcm9041117

22. Ma N, Zhang X, Zheng HT, Sun J. Shufflenet V2: Practical Guidelines for Efficient Cnn Architecture Design. ArXiv Pre-Print Server (2018) 11218:63-7. doi: 10.1007/978-3-030-01264-9_8

23. Dhalla S, Mittal A, Gupta S, Singh H. Multi-Model Ensemble to Classify Acute Lymphoblastic Leukemia in Blood Smear Images. In: Del Bimbo A, Cucchiara R, Sclaroff S, Farinella GM, Mei T, Bertini M, et al, editors. Pattern Recognition. ICPR International Workshops and Challenges. Milan, Italy: Springer International Publishing (2021). p. 243-53.

24. Feng DJ, Yi C. Computer-Aided Laboratory Animals Random Grouping. Prog Microbiol Immunol (1994) 3:35-8.

25. Peng M, Zhan W, Zhang Z, Chen T. From Macro to Micro Expression Recognition: Deep Learning on Small Datasets Using Transfer Learning. 13th IEEE International Conference on Automatic Face Gesture Recognition (2018) 11:657-61. doi: 10.1109/FG.2018.00103

26. Diederik, Ba J, Adam. A Method for Stochastic Optimization. ArXiv Pre-Print Server (2017) cs.LG:1412.6980 arXiv:1412.6980.

27. Chen S, Liu W, Qin J, Chen L, Bin G, Zhou Y, et al. Research Progress of Computer-Aided Diagnosis in Cancer Based on Deep Learning and Medical Imaging. Sheng Wu Yi Xue Gong Cheng Xue Za Zhi = J Biomed Eng = Shengwu Yixue Gongchengxue Zazhi (2017) 34:314-9. doi: 10.7507/1001-5515.201609047

28. Zhang Y, Wu Q, Chen Y, Wang Y. A Clinical Assessment of an Ultrasound Computer-Aided Diagnosis System in Differentiating Thyroid Nodules With Radiologists of Different Diagnostic Experience. Front Oncol (2020) 10:557169. doi: $10.3389 /$ fonc.2020.557169

29. Ali I, Hart GR, Gunabushanam G, Liang Y, Muhammad W, Nartowt B, et al. Lung Nodule Detection via Deep Reinforcement Learning. Front Oncol (2018) 8:108. doi: $10.3389 /$ fonc. 2018.00108

30. Peng S, Liu YH, Lv WM, Liu LZ, Zhou Q, Yang H, et al. Deep Learning-Based Artificial Intelligence Model to Assist Thyroid Nodule Diagnosis and Management: A Multicentre Diagnostic Study. Lancet Digit Health (2021) 3:E250-9. doi: 10.1016/S2589-7500(21)00041-8

31. Fujisawa Y, Otomo Y, Ogata Y, Nakamura Y, Fujita R, Ishitsuka Y, et al. DeepLearning-Based, Computer-Aided Classifier Developed With a Small Dataset of Clinical Images Surpasses Board-Certified Dermatologists in Skin Tumour Diagnosis. Br J Dermatol (2019) 180:373-81. doi: 10.1111/bjd.16924

32. Shakarami A, Menhaj MB, Tarrah H. Diagnosing Covid-19 Disease Using an Efficient Cad System. Optik (2021) 241:167199. doi: 10.1016/ j.ijleo.2021.167199

33. Deepak S, Ameer PM. Brain Tumor Classification Using Deep Cnn Features via Transfer Learning. Comput Biol Med (2019) 103345:122-38. doi: 10.1016/ j.compbiomed.2019.103345

34. Liu Y, Xu Y, Meng X, Wang X, Bai T. A Study on the Auxiliary Diagnosis of Thyroid Disease Images Based on Multiple Dimensional Deep Learning Algorithms. Curr Med Imaging (2020) 16:199-205. doi: 10.2174/ 1573405615666190115155223

35. Ma L, Ma C, Liu Y, Wang X, Xie W. Diagnosis of Thyroid Diseases Using Spect Images Based on Convolutional Neural Network. J Med Imaging Health Inf (2018) 8:1684-9. doi: 10.1166/jmihi.2018.2493

36. Ma L, Ma C, Liu Y, Wang X. Thyroid Diagnosis From Spect Images Using Convolutional Neural Network With Optimization. Comput Intell Neurosci (2019) 2019:6212759. doi: 10.1155/2019/6212759

Conflict of Interest: The authors declare that the research was conducted in the absence of any commercial or financial relationships that could be construed as a potential conflict of interest.

Publisher's Note: All claims expressed in this article are solely those of the authors and do not necessarily represent those of their affiliated organizations, or those of the publisher, the editors and the reviewers. Any product that may be evaluated in 
this article, or claim that may be made by its manufacturer, is not guaranteed or endorsed by the publisher.

Copyright (๑) 2021 Guo, Xu, Li, Zheng, Pan, Qiu, Mao, Huang and Yang. This is an open-access article distributed under the terms of the Creative Commons Attribution
License (CC BY). The use, distribution or reproduction in other forums is permitted, provided the original author(s) and the copyright owner(s) are credited and that the original publication in this journal is cited, in accordance with accepted academic practice. No use, distribution or reproduction is permitted which does not comply with these terms. 